\title{
Diferenças entre medidas autorreferidas e laboratoriais de diabetes, doença renal crônica e hipercolesterolemia
}

\author{
Differences between self-reported and laboratory measures \\ of diabetes, chronic kidney disease, and hypercholesterolemia
}

Pedro Cisalpino Pinheiro (https://orcid.org/0000-0002-6954-1708) ${ }^{1}$

Marilisa Berti de Azevedo Barros (https://orcid.org/0000-0003-3974-195X) ${ }^{2}$

Célia Landmann Szwarcwald (https://orcid.org/0000-0002-7798-2095) ${ }^{3}$

Ísis Eloah Machado (http://orcid.org/0000-0002-4678-2074) ${ }^{4}$

Deborah Carvalho Malta (http://orcid.org/0000-0002-8214-5734) ${ }^{5}$
${ }^{1}$ Faculdade de Medicina, Universidade Federal de Minas Gerais (UFMG). Av Professor Alfredo Balena 190, Santa Efigênia. 30130-100 Belo Horizonte MG Brasil.

pedrocisalpino@gmail.com ${ }^{2}$ Faculdade de Ciências

Médicas, Unicamp.

Campinas SP Brasil.

${ }^{3}$ Instituto de Comunicação e Informação Científica

e Tecnológica em Saúde,

Fiocruz. Rio de Janeiro RJ

Brasil.

${ }^{4}$ Departamento de Medicina

de Família, Saúde Mental

e Coletiva, Universidade

Federal de Ouro Preto. Ouro

Preto MG Brasil.

${ }^{5}$ Escola de Enfermagem,

UFMG. Belo Horizonte MG

Brasil.

\begin{abstract}
This paper aims to compare the self-reported prevalence measured by laboratory tests and the false positive and negative values for diabetes, chronic kidney disease, and hypercholesterolemia. We used information from the interview and laboratory tests of the National Health Survey (2013, 2014-2015). Sensitivity and specificity were calculated by gender, age, schooling, having health insurance, and time since the last medical visit. We used logistic regression to analyze associated factors with false positives and negatives. Sensitivity was higher for diabetes and among older adults and those who had a medical visit more recently. Specificity was high for all diseases, with better performance among younger people, those with high schooling, and a visit more than one year ago. The likelihood of false positives and negatives decreased with schooling and increased with age. Low sensitivity suggests that prevalence might be higher than indicated by self-reported measures.
\end{abstract}

Key words Sensitivity and specificity, Self-report, Diabetes Mellitus, Chronic kidney disease, Hypercholesterolemia
Resumo O objetivo deste artigo é comparar as prevalências autorreferidas e medidas por exames laboratoriais, assim como a ocorrência de valores de falsos positivos e negativos, para diabetes, doença renal crônica e hipercolesterolemia. Foram utilizadas informações da entrevista e exames laboratoriais da Pesquisa Nacional de Saúde (2013, 2014-2015). Foram calculadas a sensibilidade e a especificidade, segundo sexo, idade, escolaridade, ter plano de saúde e tempo desde a última consulta médica. Por meio de regressão logística, foram analisados fatores associados à ocorrência de falsos positivos e falsos negativos. A sensibilidade foi mais elevada para o diabetes e entre os idosos e os que tiveram consulta médica mais recentemente. A especificidade foi alta para todas as doenças, com melhor desempenho entre os jovens, os com alta escolaridade e os que consultaram há mais de um ano. As chances de falsos positivos e falsos negativos diminuíram com a escolaridade e aumentaram com a idade. A sensibilidade baixa indica que as prevalências podem ser mais elevadas que as medidas autoreferidas apontam.

Palavras-chave Sensibilidade e especificidade, Autorrelato, Diabetes Mellitus, Insuficiência renal crônica, Hipercolesterolemia 


\section{Introdução}

A medida autorreferida de saúde é utilizada frequentemente como alternativa aos inquéritos que, de modo geral, demandam um processo mais complexo para a coleta de dados, além de representar custos mais elevados ${ }^{1}$. Moreira et al. ${ }^{1}$, no entanto, salientam que as prevalências autorreferidas (muitas vezes presentes em pesquisas domiciliares) podem apresentar viés. Os entrevistados podem se classificar como doentes e não apresentar a doença (falso-positivo), ou não declarar a doença e ser diagnosticado com a mesma (falso-negativo).

Há uma série de fatores que podem explicar a falta de compreensão sobre a própria situação de saúde. Johnston et al. ${ }^{2}$, por exemplo, destacam: os indivíduos não reconhecem condição que se encontram; eles podem ter fornecido informações incorretas para os médicos; podem ter esquecido ou ter interpretado equivocadamente o conselho médico; ou podem ter recebido informações incorretas do profissional. Os mesmos autores salientam que a falta de compreensão sobre a própria condição de saúde pode variar de acordo com o nível socioeconômico. Assim, a análise das condições de saúde autorreferidas será, provavelmente, influenciada por fatores como renda e educação ${ }^{3}$.

Segundo Velakkal et al. ${ }^{4}$, estudos epidemiológicos de doenças crônicas não transmissíveis (DCNT) em países em desenvolvimento tendem a mostrar menores prevalências nos grupos de status socioeconômicos mais baixo, principalmente em função da dificuldade de acesso aos serviços de saúde. Na Índia, os autores compararam o diagnóstico autorreferido com o baseado em testes padrão (baseados em questionários estruturados para a identificação de algumas enfermidades) para algumas DCNT e identificaram importantes diferenças. Os diagnósticos autorreferidos eram mais prevalentes nos grupos de maior renda e de maior escolaridade. Contudo, quando se utilizou o teste padrão, as diferenças não se mantiveram. O que, na visão dos autores, pode ser associado à dificuldade de acesso aos serviços de saúde dos grupos em pior situação socioeconômica. Mesmo que sejam capazes de identificar a pior condição de saúde em que se encontram, muitas vezes não conseguem acessar os serviços.

No Brasil, considerando a população acima de 18 anos, a prevalência da diabetes mellitus (DM) autorreferida era mais elevada que a diagnosticada com base em exame laboratorial, de acordo com os dados da PNS (2014-2015)5. A diferença entre as prevalências de DM foi maior para o grupo de 60 anos e mais, sendo a medida autorreferida mais prevalente. No grupo entre 35 e 44 anos, os diagnósticos laboratoriais indicaram prevalência superior à autorreferida. Em relação à doença renal crônica (DRC), as estimativas laboratoriais (PNS 2014-2015) indicam que o número de indivíduos com a doença é cerca de quatro vezes mais elevado que o número autorreferido ${ }^{6}$. No que diz respeito ao colesterol, a situação é bastante similar à observada com a DRC: a prevalência medida pelos exames laboratoriais é consideravelmente superior ao que os diagnósticos autorreferidos apontam ${ }^{7}$.

Diabetes, colesterol elevado e DRC apresentam diferentes manifestações sintomáticas e clínicas, o que implica em chances distintas de identificação por parte dos indivíduos ${ }^{8-10}$. O perfil da patologia, além de características sociodemográficas e de acesso aos serviços de saúde, influencia em que medida as pessoas têm consciência sobre a própria saúde $e^{8-10}$. No entanto, a análise conjun$\mathrm{ta}$ - para diferentes enfermidades - das diferenças entre diagnósticos autorreferidos e medidos laboratorialmente pode nos auxiliar a melhor dimensionar a qualidade da informação autorreferida de saúde, bem como as diferenças entre os desfechos. A análise estratificada por diferentes variáveis sociodemográficas pode, também, ser de grande importância para o planejamento de políticas públicas de saúde, ao apontar eventuais grupos mais sensíveis. A Pesquisa Nacional de Saúde (PNS), de abrangência nacional, contém uma série de marcadores biológicos que viabilizam a realização da proposta.

Neste contexto, o objetivo do presente trabalho é comparar a prevalência entre o diagnóstico autorreferido - coletado na primeira etapa da Pesquisa Nacional de Saúde (PNS), em 2013 - e $o$ aferido por meio de exames laboratoriais na segunda etapa da pesquisa, entre 2014 e 2015, com base na sensibilidade e especificidade, para a diabetes, a doença renal crônica e o colesterol elevado. Estas medidas serão analisadas considerando características sociodemográficas e de acesso a serviços de saúde. Pretende-se analisar, também, como essas características influenciam as chances de diagnósticos falso positivos e falso negativos.

\section{Métodos}

Trata-se de estudo transversal utilizando como fonte informações as duas etapas da Pesquisa Na- 
cional de Saúde (PNS). Os dados autorreferidos foram construídos a partir da resposta à pergunta se algum médico já o/a havia diagnosticado com algumas das enfermidades de interesse. No modulo de doenças crônicas da PNS, coletado em 2013, há uma questão específica para cada um dos desfechos: Diabetes (Q030); Colesterol (Q060); Doença Renal Crônica (Q124). Esses resultados foram comparados com os exames laboratoriais de Hemoglobina Glicosilada (HG), Creatinina, Taxa de Filtração Glomerular, Colesterol Total e de Frações (LDL, HDL e CT), realizados nos mesmos indivíduos entre 2014 e 2015.

A base com exames laboratoriais da PNS é constituída de 8.952 observações, baseada em uma subamostra da PNS. A construção da base laboratorial da PNS pode ser melhor compreendida em outros trabalhos ${ }^{5,11-13}$. Em função de perdas na amostra laboratorial, no processamento das análises, em material insuficiente e em outros, o número de exame aqui analisados variou entre 7.211 a 8.528. A amostra considerada para cada um dos desfechos, assim como para cada uma das diferentes métricas, contava com observações com as informações autorreferidas e laboratoriais. Ou seja, foram desconsiderados os casos com valores missing em alguma dessas variáveis.

O diagnóstico laboratorial de diabetes foi baseado na Hemoglobina Glicosilada (HgA1), para indivíduos com HgA1 maior ou igual a 6,5. Este ponto de corte foi utilizado em trabalho recentemente publicado com a mesma base de dados ${ }^{5}$. Convém destacar que foram excluídos da análise sobre diabetes os casos em que os indivíduos apresentavam HgAl menor ou igual a 6.5 (que representaria ausência de diabetes), mas que declaram ter utilizado medicamento para baixar o açúcar ou insulina nas duas semanas anteriores à realização da pesquisa (Q03401 ou Q03402). A manutenção desses casos na base poderia inflar artificialmente os casos falso positivo e a especificidade. O mesmo exercício, em razão da disponibilidade de informações, não pôde ser realizado para os demais desfechos.

Para a Doença Renal Crônica (DRC), duas diferentes métricas foram utilizadas: a taxa de filtração glomerular (TFG) e a creatinina (CR). A definição dos pontos de corte seguiu o trabalho de Malta et al. ${ }^{6}$, sendo o diagnóstico de DRC atribuído para os homens com CR igual ou superior a 1,3 e de 1,1 para as mulheres. Com base na TFG, foram considerados com insuficiência renal crônica todos aqueles com valores inferiores a 60 $\mathrm{mL} / \mathrm{min} / 1,73 \mathrm{~m}^{2}$. O cálculo da TFG na base de dados foi baseado na equação do estudo MDRD sem correção de raça e cor $^{14}$. Três diferentes métricas e pontos de corte foram utilizados para a definição do diagnóstico de colesterol alterado: colesterol total $\geq 200 \mathrm{mg} / \mathrm{dL}$; lipoproteínas de baixa densidade (LDL) $\geq 130 \mathrm{mg} / \mathrm{dL}$; e lipoproteínas de alta densidade (HDL) $<40 \mathrm{mg} / \mathrm{dL}$ para homens e $<50 \mathrm{mg} / \mathrm{dL}$ para mulheres. Convém destacar que a pergunta utilizada para a identificação autorreferida faz referência ao colesterol alto, e não alterado, o que poderia levar a alguma eventual confusão relacionada ao HDL. A definição dos limites foi baseada em estudo com a mesma base de dados ${ }^{7}$ e na diretriz brasileira de dislipidemia ${ }^{15}$.

A primeira estratégia para a análise das diferenças entre os diagnósticos medidos laboratorialmente e autorreferidos foi a avaliação da sensibilidade e da especificidade para cada um dos desfechos de interesse, considerando o conjunto de variáveis sociodemográficas - sexo, idade, escolaridade, possuir ou não plano de saúde e o tempo passado desde a última consulta médica. A sensibilidade indica a proporção de indivíduos que declararam possuir uma das enfermidades entre aqueles que o exame laboratorial indicava de fato a presença da doença. Por sua vez, a especificidade representa a proporção dos indivíduos que responderam não possuir a doença e o exame confirmava ausência da mesma ${ }^{16}$. Ou seja, indica a proporção dos casos em que o diagnostico autorreferido negativo foi referendado pela medida laboratorial. Na análise, os resultados da base laboratorial foram tratados como padrão ouro. As sensibilidade e especificidade de cada uma das categorias das variáveis de interesse, para cada desfecho e métrica, foram calculadas separadamente. Assim, foram gerados intervalos de confinação (IC 95\%) para cada subgrupo.

Em seguida, foram elaborados modelos de regressão logística para duas variáveis dependentes (para cada desfecho), os casos falsos positivos e os falsos negativos. Ou seja, para a diabetes, a DRC e o colesterol, à variável falso positivo foi atribuído valor 01 (um), nos casos em que os indivíduos declararam presença da doença, mas a informação não foi corroborada pelo exame laboratorial, e zero para os demais casos. Recebeu valor 01 (um) na variável falso negativo os indivíduos em que o diagnóstico laboratorial apontou a presença da doença e os entrevistados não o fizeram, aos demais casos foi atribuído valor zero. Como variáveis explicativas foram utilizadas as mesmas variáveis sociodemográficas e de acesso aos serviços de saúde, já descritas na análi- 
se de sensibilidade e especificidade. $O$ modelo foi elaborado para os três desfechos considerando cada uma das métricas utilizadas (HgAl, TFG, CR, CT, LDL e HDL).

A PNS 2013 foi aprovada pela Comissão Nacional de Ética em Pesquisa (CONEP) do Conselho Nacional de Saúde, Ministério da Saúde. Os participantes da pesquisa assinaram o termo de consentimento livre e esclarecido (TCLE) e posteriormente foram realizadas coletas de sangue periférico a qualquer hora do dia. Todas as estimativas e análises foram elaboradas utilizando o software Data Analysis and Statistical Software (Stata), versão 2014.

\section{Resultados}

\section{Diabetes}

A prevalência do diabetes medida com base na Hemoglobina Glicosilada (HgA1), na amostra aqui analisada, foi de 7,4\% (IC95\%, 6,7\%-8,2\%), enquanto que a informação autorreferida aponta para uma prevalência de 5,5 (IC95\%, 4,9\%$6,3 \%)$. A diferença entre as prevalências apresentou significância estatística. A Tabela 1 mostra que 59\% (IC95\%, 53,8\%-63,4\%) dos indivíduos com diagnóstico laboratorial de diabetes declararam apresentar a doença.

A sensibilidade em relação ao diabetes de homens e mulheres foi praticamente a mesma, respectivamente, 58,9\% (IC95\%, 50,5\%-66,9\%) e 59\% (IC95\%, 52,4\%-65,2\%). Considerando os grupos de idade, a sensibilidade em relação ao diabetes foi mais baixa no grupo mais jovem (menor de 50 anos), a diferença entre este grupo e os demais (50-59 e 60 e mais) foi estatisticamente significativa. Os níveis de escolaridade não apresentaram diferenças significativas para a sensibilidade relacionada ao diabetes.

Indivíduos que haviam visitado o médico em um período superior a um ano, com diagnóstico laboratorial de diabetes, reportavam a presença da doença (26,8\% - IC95\%, 17,0\%-39,5\%) em níveis mais baixos que os grupos cuja consulta médica se deu mais recentemente (ambos acima de $60 \%$ de sensibilidade). Possuir plano de saúde não representou diferença significativa relacionada à sensibilidade para as medidas laboratoriais e autorreferidas de diabetes.

A Tabela 2 mostra a especificidade das medidas autorreportadas e baseada em exames laboratoriais para todos os desfechos. Para o diabetes, 98,8\% (IC95\%, 98,4\%-99,1\%) dos indivíduos com Hemoglobina Glicosilada abaixo de 6,5\% declararam não apresentar a doença. De modo geral, a especificidade do diabetes foi bastante elevada. Entre as variáveis aqui analisadas, apenas a idade apresentou diferença significativa entre os subgrupos. A especificidade para os menores de 50 anos (99,3\% IC95\%, 98,9\%-99,6\%) foi mais alta que entre aqueles com 60 anos ou mais $(97 \%)$.

As Tabelas 3 e 4 mostram, respectivamente, para cada desfecho, a razão de chance (Odds Ratio - OR) estimada por meio de modelo de regressão logística para duas variáveis dependentes; os casos de falso negativo (FN) e os de falso positivo (FP). Considerando o diabetes, a chance de falso negativo aumentou de modo significativo com a idade. Em relação ao grupo com 50 anos ou menos, tanto os indivíduos entre 50 e 59 anos $(\mathrm{OR}=2)$ quanto aqueles maiores de 60 anos (OR $=3,18$ ) apresentavam chances mais elevadas de FN. Em relação à escolaridade, o modelo mostra que indivíduos que haviam acessado o nível superior apresentavam chance $51 \%(\mathrm{OR}=0,49)$ mais baixa de registro de falso negativo, em relação ao grupo sem instrução ou com ensino fundamental incompleto. As demais variáveis (sexo, última consulta e plano de saúde) não apontaram significância estatística no modelo estimado para analisar a ocorrência de FN para o diabetes.

A Tabela 4 indica que ser maior de 60 anos, em relação ao grupo com menos de 50, aumentava significativamente $(183 \%, \mathrm{OR}=2,83)$ a chance de ocorrência de falso positivo para o diabetes. As demais variáveis não apresentaram significância estatística.

\section{Doença Renal Crônica}

A prevalência da doença renal crônica baseada na Creatinina foi de 5\% (IC95\%, 4,5\%-5,6\%) e 6,6\% (IC95\%, 6-7,4\%), considerando a Taxa de Filtração Glomerular. Por sua vez, a prevalência autorreportada de DRC foi de 1,4\% em ambas as amostras utilizadas para a análise dos desfechos. Tanto para a TFG como para a Creatinina, a sensibilidade das medidas laboratoriais e autorreferidas foi bastante baixa, respectivamente, 4,4\% (IC95\%, 2,7\%-7\%) e 3,2\% (IC95\%, 2,1\%-5,1\%). Ou seja, apenas uma pequena parcela dos indivíduos com diagnostico laboratorial de DRC reportaram adequadamente a presença da doença. A análise da sensibilidade da doença renal crônica (Tabela 1), considerando ambas as métricas, não apresentou diferenças significativas entre os subgrupos das variáveis analisadas. Vale destacar, 
Tabela 1. Sensibilidade do Diabetes Mellitus, DRC e colesterol (CT, HDL, LDL) autorreferida comparada com as medidas laboratoriais.

\begin{tabular}{|c|c|c|c|c|c|c|}
\hline & Diabetes & $\begin{array}{c}\text { Doença Renal } \\
\text { Crônica } \\
\text { (Creatinina) }\end{array}$ & $\begin{array}{c}\text { Doença Renal } \\
\text { Crônica } \\
\text { (TFG) }\end{array}$ & $\begin{array}{l}\text { Colesterol } \\
\text { Total }\end{array}$ & $\begin{array}{l}\text { Colesterol } \\
\text { LDL }\end{array}$ & $\begin{array}{c}\text { Colesterol } \\
\text { HDL }\end{array}$ \\
\hline Total & 59 & 4.4 & 3.2 & 24.2 & 27.4 & 16.5 \\
\hline IC 95\% & $(53.8 ; 63.9)$ & $(2.7 ; 7)$ & $(2.1 ; 5.1)$ & $(22.2 ; 26.3)$ & $(24.6 ; 30.3)$ & $(15.1 ; 17.2)$ \\
\hline N.Obs. & 7347 & 8528 & 7450 & 7222 & 7222 & 7211 \\
\hline \multicolumn{7}{|l|}{ Sexo } \\
\hline Mulher & 59 & 3.7 & 2.6 & 27.1 & 30.3 & 18.5 \\
\hline IC 95\% & $(52.4 ; 65.2)$ & $(1.9 ; 7.3)$ & $(1.3 ; 5)$ & $(24.6 ; 29.9)$ & $(26.7 ; 34)$ & $(16.6 ; 21.4)$ \\
\hline N.Obs. & 4469 & 4980 & 4340 & 4428 & 4427 & 4422 \\
\hline Homens & 58.9 & 5 & 4.4 & 19.8 & 23.3 & 13.4 \\
\hline IC 95\% & $(50.5 ; 66.9)$ & $(2.6 ; 9.4)$ & $(2.4 ; 8.1)$ & $(16.8 ; 23.3)$ & $(19 ; 28.1)$ & $(11.2 ; 15.9)$ \\
\hline N.Obs. & 2878 & 3548 & 3110 & 2794 & 2795 & 2789 \\
\hline \multicolumn{7}{|l|}{ Idade } \\
\hline Menor 50 & 42.2 & 2.8 & 3.3 & 16.5 & 18 & 10.5 \\
\hline IC 95\% & $(31.1 ; 54)$ & $(0.9 ; 7.9)$ & $(1.2 ; 8.8)$ & $(14 ; 19.4)$ & $(14.5 ; 22)$ & $(8.9 ; 13.1)$ \\
\hline N.Obs. & 4238 & 5019 & 4756 & 4029 & 4028 & 4022 \\
\hline $50-59$ & 65.9 & 5 & 2.4 & 27.8 & 33.5 & 21.3 \\
\hline IC 95\% & $(56.2 ; 74.4)$ & $(2 ; 12.1)$ & $(1 ; 5.8)$ & $(23.6 ; 32.5)$ & $(27.6 ; 39.9)$ & $(17.8 ; 25.2)$ \\
\hline N.Obs. & 1314 & 1495 & 1419 & 1318 & 1318 & 1318 \\
\hline 60 ou mais & $64(57 ; 70.5)$ & 5.4 & 3.7 & 33 & 36.4 & 26.7 \\
\hline IC 95\% & $(57 ; 70.5)$ & $(2.9 ; 9.9)$ & $(2 ; 6.5)$ & $(29.3 ; 37)$ & $(31.4 ; 41.7)$ & $(23.6 ; 26.7)$ \\
\hline N.Obs. & 1795 & 2014 & 1275 & 1875 & 1876 & 1871 \\
\hline \multicolumn{7}{|l|}{ Esc. } \\
\hline S.Instrução ou F. Incompleto & 61.2 & 6.9 & 4.9 & 27.3 & 31.8 & 20.5 \\
\hline IC 95\% & $(54.7 ; 67.3)$ & $(3.9 ; 11.8)$ & $(2.8 ; 8.3)$ & $(24.4 ; 30.5)$ & $(27.9 ; 36.1)$ & $(18.2 ; 20.6)$ \\
\hline N.Obs. & 3113 & 3785 & 3086 & 3087 & 3088 & 3079 \\
\hline $\begin{array}{l}\text { F. Completo ou M. } \\
\text { Completo }\end{array}$ & 54.6 & 1.8 & 1.3 & 21 & 23.2 & 13.5 \\
\hline IC 95\% & $(45 ; 63.8)$ & $(0.8 ; 4.3)$ & $(0.5 ; 3.2)$ & $(18 ; 24.5)$ & $(19 ; 28)$ & $(11.4 ; 16)$ \\
\hline N.Obs. & 3079 & 3499 & 3217 & 2982 & 2981 & 2979 \\
\hline Superior & 62.1 & 1.9 & 2 & 23.8 & 26 & 14.9 \\
\hline IC 95\% & $(44.6 ; 77)$ & $(0.4 ; 8.3)$ & $(0.6 ; 6.7)$ & $(19.1 ; 29.3)$ & $(19.2 ; 34.1)$ & $(11.7 ; 21.3)$ \\
\hline N.Obs. & 1155 & 1244 & 1147 & 1153 & 1153 & 1153 \\
\hline \multicolumn{7}{|l|}{ Última consulta } \\
\hline Menos de 3 meses & 64.4 & 5.9 & 3.4 & 27.8 & 31.7 & 19.7 \\
\hline IC 95\% & $(57.8 ; 70.5)$ & $(3.5 ; 10)$ & $(1.9 ; 6)$ & $(25 ; 30.8)$ & $(27.9 ; 35.9)$ & $(17.7 ; 20.9)$ \\
\hline N.Obs. & 3958 & 4437 & 3790 & 3970 & 3971 & 3961 \\
\hline Entre 3 meses e 1 ano & 60.8 & 1.6 & 3 & 26.4 & 30.5 & 17.1 \\
\hline IC 95\% & $(50.4 ; 70.3)$ & $(0.4 ; 6.2)$ & $(1.1 ; 7.9)$ & $(22.2 ; 31.1)$ & $(24.6 ; 37.1)$ & $(14.2 ; 19.8)$ \\
\hline N.Obs. & 1770 & 1982 & 1755 & 1733 & 1732 & 1733 \\
\hline Mais de 1 ano & 26.8 & 2.1 & 2.9 & 10.8 & 11.9 & 6.7 \\
\hline IC 95\% & $(17 ; 39.5)$ & $(0.6 ; 7.3)$ & $(1.1 ; 7.7)$ & $(8.3 ; 14)$ & $(8.5 ; 16.4)$ & $(4.9 ; 11.1)$ \\
\hline N.Obs. & 1619 & 2109 & 1905 & 1519 & 1519 & 1517 \\
\hline \multicolumn{7}{|l|}{ Plano de Saúde } \\
\hline Não & 56.5 & 4.1 & 3.5 & 22.6 & 26.4 & 15.4 \\
\hline IC 95\% & $(50.5 ; 62.2)$ & $(2.3 ; 7.1)$ & $(2.1 ; 6)$ & $(20.3 ; 25)$ & $(23.3 ; 29.8)$ & $(13.8 ; 16.9)$ \\
\hline N.Obs. & 5480 & 6527 & 5732 & 5335 & 5335 & 5327 \\
\hline Sim & 65.4 & 4.9 & 2.6 & 27.8 & 29.8 & 19.1 \\
\hline IC 95\% & $(55 ; 74.5)$ & $(2.1 ; 10.9)$ & $(1.1 ; 5.9)$ & $(23.9 ; 32.1)$ & $(24.4 ; 35.9)$ & $(16.2 ; 20.8)$ \\
\hline N.Obs. & 1867 & 2001 & 1718 & 1887 & 1887 & 1884 \\
\hline
\end{tabular}

Fonte: Pesquisa Nacional de Saúde (PNS), 2013; 2014-2015. 
Tabela 2. Especificidade do Diabetes Mellitus, DRC e colesterol (CT, HDL, LDL) autorreferida comparada com as medidas laboratoriais.

\begin{tabular}{|c|c|c|c|c|c|c|}
\hline & Diabetes & $\begin{array}{c}\text { Doença } \\
\text { Renal } \\
\text { Crônica } \\
\text { (Creatinina) }\end{array}$ & $\begin{array}{l}\text { Doença } \\
\text { Renal } \\
\text { Crônica } \\
\text { (TFG) }\end{array}$ & $\begin{array}{c}\text { Colesterol } \\
\text { Total }\end{array}$ & $\begin{array}{c}\text { Colesterol } \\
\text { LDL }\end{array}$ & $\begin{array}{c}\text { Colesterol } \\
\text { HDL }\end{array}$ \\
\hline Total & 98.8 & 98.7 & 98.7 & 89.1 & 87.5 & 85.7 \\
\hline IC 95\% & $(98.4 ; 99.1)$ & $(98.3 ; 99)$ & $(98.3 ; 99)$ & $(87.9 ; 90.2)$ & $(86.4 ; 88.6)$ & $(84.2 ; 87.1)$ \\
\hline N.Obs. & 7347 & 8528 & 7450 & 7222 & 7222 & 7211 \\
\hline \multicolumn{7}{|l|}{ Sexo } \\
\hline Mulher & 98.6 & 98.5 & 98.5 & 87.7 & 85.6 & 83.3 \\
\hline IC 95\% & $(98.1 ; 99)$ & $(97.9 ; 98.9)$ & $(97.8 ; 99)$ & $(86 ; 89.2)$ & $(84 ; 87)$ & $(81.1 ; 85.2)$ \\
\hline N.Obs. & 4469 & 4980 & 4340 & 4428 & 4427 & 4422 \\
\hline Homens & 99 & 99 & 99 & 90.8 & 89.9 & 88.1 \\
\hline IC 95\% & $(98.4 ; 99.4)$ & $(98.5 ; 99.3)$ & $(98.5 ; 99.3)$ & $(89 ; 92.3)$ & $(88.2 ; 91.3)$ & $(85.9 ; 90)$ \\
\hline N.Obs. & 2878 & 3548 & 3110 & 2794 & 2795 & 2789 \\
\hline \multicolumn{7}{|l|}{ Idade } \\
\hline Menor 50 & 99.3 & 99 & 99 & 94.2 & 93 & 93.2 \\
\hline IC 95\% & $(98.9 ; 99.6)$ & $(98.5 ; 99.3)$ & $(98.5 ; 99.3)$ & $(93 ; 95.3)$ & $(91.8 ; 94)$ & $(91.6 ; 94.4)$ \\
\hline N.Obs. & 4238 & 5019 & 4756 & 4029 & 4028 & 4022 \\
\hline $50-59$ & 98.5 & 98 & 98.1 & 84.4 & 83.5 & 79.1 \\
\hline IC 95\% & $(97.5 ; 99)$ & $(96.9 ; 98.7)$ & $(97 ; 98.8)$ & $(80.5 ; 87.7)$ & $(80.2 ; 86.3)$ & $(74.6 ; 83)$ \\
\hline N.Obs. & 1314 & 1495 & 1419 & 1318 & 1318 & 1318 \\
\hline 60 ou mais & 97.3 & 98.4 & 98.3 & 76.7 & 75.4 & 71.6 \\
\hline IC 95\% & $(96.1 ; 98.1)$ & $(97.6 ; 99)$ & $(97.2 ; 99)$ & $(73.4 ; 79.7)$ & $(72.6 ; 78.1)$ & $(67.7 ; 75.1)$ \\
\hline N.Obs. & 1795 & 2014 & 1275 & 1875 & 1876 & 1871 \\
\hline \multicolumn{7}{|l|}{ Esc. } \\
\hline S.Instrução ou F. Incompleto & 98 & 98.8 & 98.9 & 85 & 83.7 & 80.8 \\
\hline IC $95 \%$ & $(97.3 ; 98.5)$ & $(98.3 ; 99.1)$ & $(98.5 ; 99.2)$ & $(82.8 ; 86.9)$ & $(81.8 ; 85.5)$ & $(78.2 ; 83.2)$ \\
\hline N.Obs. & 3113 & 3785 & 3086 & 3087 & 3088 & 3079 \\
\hline F. Completo ou M. Completo & 99.2 & 98.4 & 98.4 & 92.5 & 90.7 & 90 \\
\hline IC $95 \%$ & $(98.5 ; 99.5)$ & $(97.7 ; 98.9)$ & $(97.7 ; 99)$ & $(90.8 ; 93.9)$ & $(89.1 ; 92.1)$ & $(87.8 ; 91.8)$ \\
\hline N.Obs. & 3079 & 3499 & 3217 & 2982 & 2981 & 2979 \\
\hline Superior & 99.3 & 99.3 & 99.2 & 88.8 & 87.2 & 85 \\
\hline IC 95\% & $(98.6 ; 99.7)$ & $(98.5 ; 99.7)$ & $(98.2 ; 99.7)$ & $(85.6 ; 91.3)$ & $(84.3 ; 89.5)$ & $(81.2 ; 88.2)$ \\
\hline N.Obs. & 1155 & 1244 & 1147 & 1153 & 1153 & 1153 \\
\hline \multicolumn{7}{|l|}{ Última consulta } \\
\hline Menos de 3 meses & 98.4 & 98.5 & 98.5 & 86.3 & 84.6 & 82.6 \\
\hline IC 95\% & $(97.9 ; 98.8)$ & $(97.9 ; 98.9)$ & $(97.8 ; 98.9)$ & $(84.5 ; 87.9)$ & $(83 ; 86.1)$ & $(80.4 ; 84.7)$ \\
\hline N.Obs. & 3958 & 4437 & 3790 & 3970 & 3971 & 3961 \\
\hline Entre 3 meses e 1 ano & 99.2 & 98.7 & 98.8 & 89.6 & 87.6 & 85.2 \\
\hline IC $95 \%$ & $(98.6 ; 99.6)$ & $(97.9 ; 99.2)$ & $(98 ; 99.3)$ & $(87.1 ; 91.7)$ & $(85.2 ; 89.6)$ & $(81.7 ; 88)$ \\
\hline N.Obs. & 1770 & 1982 & 1755 & 1733 & 1732 & 1733 \\
\hline Mais de 1 ano & 99.2 & 99.2 & 99.2 & 96.3 & 95.4 & 94.7 \\
\hline IC 95\% & $(98.1 ; 99.7)$ & $(98.5 ; 99.6)$ & $(98.4 ; 99.6)$ & $(94.5 ; 97.5)$ & $(93.8 ; 96.6)$ & $(92.7 ; 96.2)$ \\
\hline N.Obs. & 1619 & 2109 & 1905 & 1519 & 1519 & 1517 \\
\hline \multicolumn{7}{|l|}{ Plano de Saúde } \\
\hline Não & 98.6 & 98.8 & 98.9 & 90.3 & 88.9 & 87.3 \\
\hline IC 95\% & $(98.1 ; 99)$ & $(98.4 ; 99.1)$ & $(98.5 ; 99.2)$ & $(88.9 ; 91.5)$ & $(87.7 ; 90.1)$ & $(85.6 ; 88.8)$ \\
\hline N.Obs. & 5480 & 6527 & 5732 & 5335 & 5335 & 5327 \\
\hline Sim & 99.2 & 98.5 & 98.4 & 86.8 & 84.7 & 82.9 \\
\hline IC 95\% & $(98.6 ; 99.5)$ & $(97.5 ; 99.1)$ & $(97.3 ; 99.1)$ & $(84.3 ; 88.9)$ & $(82.4 ; 86.7)$ & $(79.9 ; 85.6)$ \\
\hline N.Obs. & 1867 & 2001 & 1718 & 1887 & 1887 & 1884 \\
\hline
\end{tabular}

Fonte: Pesquisa Nacional de Saúde (PNS), 2013; 2014-2015. 
Tabela 3. Razão de Chances estimada para ocorrência de Falso Negativo, por variáveis socioeconômicas, para cada desfecho analisado, PNS 2013,2014-2015.

\begin{tabular}{|c|c|c|c|c|c|c|}
\hline & Diabetes & $\begin{array}{c}\text { Doença } \\
\text { Renal } \\
\text { Crônica } \\
\text { (Creatinina) }\end{array}$ & $\begin{array}{l}\text { Doença } \\
\text { Renal } \\
\text { Crônica } \\
\text { (TFG) }\end{array}$ & $\begin{array}{c}\text { Colesterol } \\
\text { Total }\end{array}$ & $\begin{array}{c}\text { Colesterol } \\
\text { LDL }\end{array}$ & $\begin{array}{c}\text { Colesterol } \\
\text { HDL }\end{array}$ \\
\hline $\mathrm{N}^{\circ}$ Obs. & 7,347 & 8,528 & 7,450 & 7,222 & 7,222 & 7,211 \\
\hline \multicolumn{7}{|l|}{ Sexo } \\
\hline Homens & - & - & - & - & - & - \\
\hline Mulheres & 1.3 & 0.79 & $1.65^{\star}$ & 1.15 & 1.11 & $1.39^{*}$ \\
\hline \multicolumn{7}{|l|}{ Idade } \\
\hline menor 50 & - & - & - & - & - & - \\
\hline $50-59$ & $2^{\star}$ & 1.3 & $3.35^{\star}$ & $1.69^{*}$ & $1.58^{\star}$ & $0.8^{\star}$ \\
\hline maior 60 & $3.18^{\star}$ & $3.63^{\star}$ & $6.95^{\star}$ & $1.41^{\star}$ & $1.37^{\star}$ & $0.8^{*}$ \\
\hline \multicolumn{7}{|l|}{ Escolaridade } \\
\hline $\begin{array}{l}\text { Sem Instrução/Fund. } \\
\text { Incompleto }\end{array}$ & - & - & - & - & - & - \\
\hline $\begin{array}{l}\text { Fund. Comp. ou Médio } \\
\text { Completo }\end{array}$ & 0.92 & 0.95 & 0.83 & 0.99 & 1.01 & 1.02 \\
\hline Superior ou + & $0.49^{*}$ & 0.75 & 0.83 & 0.91 & 0.89 & 0.82 \\
\hline \multicolumn{7}{|l|}{ Última consulta } \\
\hline Menos de 3 meses & - & - & - & - & - & - \\
\hline entre 3 meses e 1 ano & 1.15 & 0.91 & 0.82 & 1.11 & 1.07 & 1.1 \\
\hline 1 ano ou mais & 1.18 & 0.75 & $0.73^{\star}$ & $1.31^{\star}$ & $1.41^{\star}$ & $1.21^{\star}$ \\
\hline \multicolumn{7}{|l|}{ Plano de Saúde } \\
\hline Sim & 0.78 & $1.4^{*}$ & 1.2 & 0.89 & 0.88 & $0.75^{\star}$ \\
\hline Não & - & - & - & - & - & - \\
\hline
\end{tabular}

Fonte: Pesquisa Nacional de Saúde (PNS), 2013; 2014-2015.

no entanto, os baixos níveis da sensibilidade observada para todos os subgrupos.

Quanto à especificidade, a Tabela 2 mostra valores elevados (98,7\%, IC95\%, 98,3\%-99\%) para ambas as métricas (Creatinina e TFG). Em conjunto com diabetes, foi a especificidade mais elevada observada entre os desfechos. Assim como na análise da sensibilidade, não foram identificadas diferenças significativas entre os subgrupos das variáveis sociodemográficas e de acesso aos serviços de saúde.

A Tabela 3 mostra que, em relação ao grupo menor de 50 anos, a chance de FN era maior para o grupo com mais de 60 anos $(\mathrm{OR}=3,63)$, considerando a Creatinina como medida laboratorial. No mesmo modelo, possuir plano de saúde aumentava em $40 \%(\mathrm{OR}=1,4)$ a chance de FN para DRC. A análise da ocorrência de FN para a DRC - quando a TFG foi a métrica utilizada - aponta para uma chance mais elevada de $\mathrm{FN}$ para as mulheres $(\mathrm{OR}=1,65)$. Tanto o grupo entre 50 e 59 anos $(\mathrm{OR}=3,35)$ quanto aquele com 60 anos ou mais $(\mathrm{OR}=6,95)$ apresentavam chances mais al- tas dos indivíduos declararem não estar doentes e a afirmação divergir da TFG. O mesmo modelo indicou chance mais baixa de falso negativo (OR $=0,73$ ) para aqueles que estiveram em uma consulta médica em um período superior a um ano, em relação ao grupo cuja visita ocorreu em um período inferior a três meses.

A Tabela 4 indica diferenças estatisticamente significativas nas chances de falso positivo apenas para o grupo entre 50 e 59 anos, em relação àqueles com menos de 50 anos, e apenas no modelo baseado na Creatinina. $\mathrm{Na}$ análise baseada na TFG, nenhuma das variáveis explicativas apontou significância estatística.

\section{Colesterol}

A base laboratorial mostra colesterol acima do recomendado para 33,9\% (IC95\%, 32,4\%$35,3 \%$ ) e $19,5 \%$ (IC95\%, 18,3\%-20,7\%) considerando o colesterol total (CT) e o LDL, respectivamente. Por sua vez, os exames indicam que $49,8 \%(48,3 \%-51,4 \%)$ da amostra apresentava 
Tabela 4. Razão de Chances estimada para ocorrência de Falso Positivo, por variáveis socioeconômicas, para cada desfecho analisado, PNS 2013,2014-2015.

\begin{tabular}{|c|c|c|c|c|c|c|}
\hline & Diabetes & $\begin{array}{c}\text { Doença } \\
\text { Renal } \\
\text { Crônica } \\
\text { (Creatinina) }\end{array}$ & $\begin{array}{c}\text { Doença } \\
\text { Renal } \\
\text { Crônica } \\
\text { (TFG) }\end{array}$ & $\begin{array}{l}\text { Colesterol } \\
\text { Total }\end{array}$ & $\begin{array}{l}\text { Colesterol } \\
\text { LDL }\end{array}$ & $\begin{array}{l}\text { Colesterol } \\
\text { HDL }\end{array}$ \\
\hline $\mathrm{N}^{\circ}$ Obs. & 7,347 & 8,528 & 7,450 & 7,222 & 7,222 & 7,211 \\
\hline \multicolumn{7}{|l|}{ Sexo } \\
\hline Homens & - & - & - & - & - & - \\
\hline Mulheres & 1.27 & 1.36 & 1.32 & 1.14 & $1.32^{\star}$ & $1.05^{\star}$ \\
\hline \multicolumn{7}{|l|}{ Idade } \\
\hline menor 50 & - & - & - & - & - & - \\
\hline $50-59$ & 1.92 & $1.97^{\star}$ & 1.76 & $1.97^{\star}$ & $2.04^{\star}$ & $3.17^{\star}$ \\
\hline maior 60 & $2.83^{*}$ & 1.47 & 1.46 & $2.98^{\star}$ & $3.15^{\star}$ & $3.93^{*}$ \\
\hline \multicolumn{7}{|l|}{ Escolaridade } \\
\hline $\begin{array}{l}\text { Sem Instrução/Fund. } \\
\text { Incompleto }\end{array}$ & - & - & - & - & - & - \\
\hline $\begin{array}{l}\text { Fund. Comp. ou Médio } \\
\text { Completo }\end{array}$ & 0.7 & 1.46 & 1.6 & $0.73^{\star}$ & 0.8 & 0.77 \\
\hline $\begin{array}{l}\text { Superior ou }+ \\
\text { Última consulta }\end{array}$ & 0.61 & 0.58 & 0.69 & 1.02 & 1.02 & 1.21 \\
\hline Menos de 3 meses & - & - & - & - & - & - \\
\hline entre 3 meses e 1 ano & 0.54 & 0.93 & 0.84 & 0.81 & 0.89 & 0.94 \\
\hline 1 ano ou mais & 0.6 & 0.63 & 0.64 & $0.33^{\star}$ & $0.36^{\star}$ & $0.4^{*}$ \\
\hline \multicolumn{7}{|l|}{ Plano de Saúde } \\
\hline Sim & 0.66 & 1.21 & 1.36 & $1.32^{\star}$ & $1.37^{\star}$ & $1.47^{\star}$ \\
\hline Não & - & - & - & - & - & - \\
\hline
\end{tabular}

Nota: * Significância estatística ao nível de 0,95.

Fonte: Pesquisa Nacional de Saúde (PNS), 2013; 2014-2015.

HDL abaixo do recomendado. A prevalência de colesterol alto autorreportado foi de $15,4 \%$.

A sensibilidade (Tabela 1) relacionada ao colesterol foi consideravelmente mais baixa que a observada na análise do diabetes. A sensibilidade foi mais elevada para o LDL, 27,4\% (IC95\%, 24,6\%-30,3\%), seguido pelo colesterol total $(24,2 \%$, IC95\%, 22,2\%-26,3\%) e pelo HDL (16,5\%, IC95\%, 15,1\%-17,2\%). O CT e o HDL indicaram sensibilidade mais elevada para as mulheres. Nas três medidas de colesterol, a sensibilidade do grupo com menos de 50 anos era, no mínimo, a metade daqueles com 60 ou mais. Entre os subgrupos de escolaridade, não se observou significância estatística em nenhuma das três métricas de colesterol.

A Tabela 1 indica que o tempo passado desde a última consulta influencia a sensibilidade para praticamente todos os desfechos. O mesmo foi observado nas análises baseadas no CT, LDL e HDL, particularmente quando comparados os grupos que haviam consultado um médico em um período superior a um ano e o grupo que havia consultado um médico nos últimos três meses desde a entrevista.

A especificidade foi mais baixa para as três métricas do colesterol, em relação ao diabetes e à DRC, variando entre 85,7\% (IC95\%, 84,2\%$87,1 \%$ ) e $89,1 \%$ (IC95\%, 24,6\%-30,3\%) (Tabela $2)$. Entre os sexos, a especificidade foi mais elevada para os homens, medida pelo HDL e pelo LDL. A Tabela 2 monstra que o gradiente etário da especificidade apresenta o sinal inverso ao observado na sensibilidade (Tabela 1). Ou seja, a especificidade diminuiu com o aumento da idade. Para todos os desfechos, a população mais jovem (menos de 50) apresentava uma proporção mais elevada de indivíduos não doentes (laboratorialmente) que declararam não apresentar a doença, comparados ao grupo maior de 60 anos. No caso do HDL, por exemplo, a diferença absoluta da especificidade entre esses grupos foi de $21,6 \%$. 
Por sua vez, considerando a escolaridade, as diferenças só foram estatisticamente significativas entre aqueles sem instrução ou com ensino fundamental incompleto e entre aqueles com ensino fundamental ou médio completos. A especificidade foi mais elevada para os indivíduos que foram ao médico em um período superior a um ano. Medida pelo LDL, a especificidade foi mais alta para quem não possuía plano de saúde.

A chance de ocorrência de falso negativo ( $\mathrm{Ta}-$ bela 3) era mais elevada para as mulheres, quando medida pelo HDL $(\mathrm{OR}=1.39)$. Em relação ao grupo mais novo (menos de 50 anos), as chances de FN foram quase sempre positivas com o avançar da idade. Apenas para o HDL, as chances de falso negativo diminuíam com idade. Com o passar do tempo desde a última consulta, as chances de registro de FN eram mais altas para o colesterol total, LDL e HDL. Respectivamente, chances $31 \%(\mathrm{OR}=1,31), 41 \%(\mathrm{OR}=1,41)$ e $21 \%(\mathrm{OR}=$ 1,21 ) maiores ( 1 ano ou mais), em relação àqueles que haviam ido ao médico em um período inferior a três meses. Por sua vez, possuir plano de saúde diminuía as chances de FN apenas para o colesterol medido pelo HDL $(\mathrm{OR}=0,75)$.

A Tabela 4 mostra que, comparadas a dos homens, a chance de uma mulher declarar ter recebido diagnóstico de colesterol alto e apresentar LDL dentro do esperado (laboratorialmente) (FP) foi $32 \%$ mais alta $(\mathrm{OR}=1,32)$. A chance de FP para as mulheres foi também mais elevada considerando o HDL $(\mathrm{OR}=1,05)$. As chances de alguém com mais de 60 anos reportar FP foi maior para o colesterol total $(\mathrm{OR}=2,98)$, LDL $(\mathrm{OR}=3,15)$ e $\mathrm{HDL}(\mathrm{OR}=3,93)$.

Foram poucos os desfechos que em o nível de escolaridade afetou as chances FP. A chance de alguém com ensino fundamental ou médio completo (colesterol total) representar um caso de FP era $27 \%$ menor do que para aqueles sem instrução ou com fundamental incompleto. Quanto maior o tempo passado desde a última consulta, menores as chances de FP, especialmente para o grupo em que a última visita ao médico ocorreu há mais de um ano. Possuir plano de saúde indicava chances mais elevadas de ocorrência de falso positivo.

\section{Discussão}

Considerando as métricas empregadas, os resultados indicam baixa concordância entre os diagnósticos autorreportados e os medidos laboratorialmente. Os marcadores biológicos são capazes de informar sobre as condições de saúde anteriores à percepção dos sintomas por parte dos indivíduos $^{3}$. Crimmins e Vasunilashorn ${ }^{17}$ destacam que a disponibilidade de biomarcadores possibilita aos pesquisadores conhecer a situação de saúde e a efetividade dos serviços ao mesmo tempo. A diferença entre os diagnósticos autorreferidos e os medidos laboratorialmente, principalmente em relação à sensibilidade, indicam que há uma parcela importante da população que pode estar doente e que não tem conhecimento dessa condição.

Os dados laboratoriais da PNS apontam que as estimativas de doença renal crônica foram até quatro vezes mais elevadas se comparadas os dados autorreferidos ${ }^{7}$. Os resultados mostraram que a sensibilidade foi mais elevada no diabetes, cerca de $60 \%$ daqueles que declaram apresentar a doença e que tiveram o exame positivo. Para o colesterol, esta medida foi mais baixa (próxima a um quarto) e inferior a 5\% para DRC. Portanto, a baixa sensibilidade aqui identificada pode refletir o subdiagnóstico da DRC, principalmente, no país. A comparação entre as medidas de concordância entre diferentes doenças, entretanto, deve ser realizada com cautela. A acurácia de medidas autorreferidas de saúde relaciona-se - além de características socioeconômicas, demográficas e com a presença ou não de morbidades - com características da patologia ${ }^{8-10}$. A percepção de sintomas é um dos fatores relacionados ao reconhecimento das doenças ${ }^{8-10}$.

A diabetes apresentou sensibilidade mais elevada que as demais doenças. Em grande medida esse resultado pode relacionar-se à manifestação de sintomas característicos da doença, não observadas no colesterol e na DRC. Okura et al. ${ }^{10}$ observam que a diabetes não é uma doença agressiva no início de sua manifestação, mas que demanda muitas interações com o sistema de saúde para o seu controle, o que aumenta as chances de reconhecimento da enfermidade pelos indivíduos. Em comparação à DRC e ao colesterol elevado, essa pode ser uma das razões para a maior sensibilidade observada. O colesterol, ainda que permaneça por muito tempo assintomático, é mais comumente solicitado em exames de rotina, se comparado à DRC. A manifestação clínica da condição aumenta a chance de que os indivíduos a reportem?.

Harris e Schoorp ${ }^{18}$, neste sentido, destacam que, ao contrário das medidas autorreferidas, os marcadores biométricos oferecem medidas objetivas de saúde e são capazes de apontar riscos para os casos em que os sintomas não se mani- 
festam cedo e de forma consistente. O que reforça a importância de pesquisas, como a PNS, para o planejamento de ações de saúde em nível nacional.

A especificidade, ou a proporção dos indivíduos que responderam não possuir a doença e o exame que confirmava ausência da mesma, foi elevada para todas as medidas (diabetes, DRC e colesterol), com nível mais baixo no colesterol. Apresentaram especificidade mais alta os indivíduos mais jovens, com alta escolaridade e que consultaram um médico há mais de um ano. No entanto, estes mesmos grupos apresentaram prevalências mais baixas das doenças analisadas ${ }^{5-7}$.

O estudo atual confirma resultados destacados na literatura que apontam baixa sensibilidade e alta especificidade (96\%) entre as medidas aferidas e autorreferidas ${ }^{10,19-22}$. Ning et al. ${ }^{16}$, analisando diabetes e hipertensão, observaram sensibilidade mais elevada para a população mais velha. Estudo de corte de idosos em Bambuí também identificou sensibilidade para diabetes mais alta entre os que visitaram o médico mais recentemente, assim como entre aqueles que possuíam alguma escolaridade ${ }^{19,23}$. Em geral, a sensibilidade foi mais elevada entre os mais velhos.

$\mathrm{Na}$ maioria dos desfechos e das métricas considerados, o grupo que havia consultado um médico em um período inferior a três meses apresentou sensibilidade mais elevada que aqueles em que a última consulta havia ocorrido em um período superior. Maior acesso aos serviços de saúde, com maior chance de ter a doença diagnosticada, bem como a capacidade de recordar as orientações medicas e de mudar atitudes pode explicar esse resultado ${ }^{19,23}$. Outros trabalhos também apontam que o uso de serviços de saúde é o fator que mais se associa à validade da morbidade autorreferida ${ }^{17,19,24-26}$.

Quanto à escolaridade, há estudos que apontam para direções distintas. Wu et al. ${ }^{20}$, na China, identificaram maior capacidade de reportar corretamente a doença entre aqueles com maior escolaridade e com maior número de doenças crônicas. Goldman et al..$^{22}$, por sua vez, associam qualidade da informação autorreportada com melhor função cognitiva. Outro estudo na China sobre diabetes mostrou que o grupo com educação primária apresentou sensibilidade mais elevada que aquele com educação secundária ${ }^{16}$. Em contexto holandês, Molenaar et al. ${ }^{21}$ observam sensibilidade mais elevada no nível intermediário de escolaridade, em relação àqueles com ensino superior. A PNS, em relação à escolaridade, apresentou maior sensibilidade nos grupos de escolaridade mais baixa (sem instrução ou fundamental incompleto), principalmente se comparado ao nível intermediário (fundamental completo ou médio completo). De modo geral, o grupo que acessou o nível superior apresentou sensibilidade mais elevada que o intermediário, mas inferior ao primeiro grupo.

Cabe ressaltar, no entanto, que é bastante provável que este indicador sofra com o efeito da composição etária. Os níveis de escolaridade mais baixos são mais comuns na população mais idosa, mesmo grupo que apresenta sensibilidade mais elevada. Neste sentido, os resultados sofrem influência da estrutura etária dos grupos de escolaridade. Considerando apenas a população com 60 anos e mais com diabetes, para ilustrar $\mathrm{o}$ argumento, a sensibilidade mostra o gradiente esperado: 74,7\% (superior ou mais), 65,8 (Fundamental ou Médio Completo) e 62,7 (Sem Instrução ou Fundamental Incompleto). Por sua vez, em linhas gerais, a especificidade cresce junto ao nível de escolaridade, concordando com outros trabalhos ${ }^{16,21}$. Em relação ao efeito de composição na especificidade, pode-se compreender também que os grupos etários de maior escolaridade são, de modo geral, mais novos, e também o mesmo grupo de maior especificidade ${ }^{27}$.

Escolaridade e acesso a planos de saúde podem ser considerados proxys de renda. Davillas et al. ${ }^{3}$ destacam que, diferentemente das medidas autorreportadas de saúde, marcadores biológicos podem revelar associação entre doença e renda antes da enfermidade tornar-se explícita. Harris e Schorpp ${ }^{18}$ salientam que a identificação de riscos a partir de marcadores biológicos possibilita que os planejadores intervenham nos determinantes sociais de risco para a saúde antes que a doença se manifeste ou que haja agravamento do quadro.

Os modelos de regressão reforçam a impressão de que a idade é um fator extremamente importante para analisar a concordância entre diagnóstico medido e autorreferido. Tanto para o falso positivo (indivíduos sem diagnóstico laboratorial, mas que autorreportaram a presença da doença) como para falso negativo (diagnóstico laboratorial positivo, não referendado pela informação autorreportada), a chance de declarar incorretamente o diagnóstico, para todos os desfechos, aumentava de modo substancial com a idade. Este resultado é interessante à luz da sensibilidade mais elevada para os grupos de idade mais avançada. Apesar de, a população mais velha, proporcionalmente, ter maior conhecimento sobre a própria condição de saúde, apresenta também chance mais elevada de reportar equi- 
vocamente sua condição, independentemente do desfecho analisado.

$\mathrm{Na}$ literatura, essa relação pode ser identificada nos dois sentidos, Johnson et al. ${ }^{2}$, por exemplo, observam relação positiva entre a idade e a ocorrência de falso negativo para hipertensão crônica. Onur e Velamuri ${ }^{28}$, por sua vez, observam efeito negativo da idade na ocorrência de FN para hipertensão e para doença pulmonar. Convém salientar, que estes autores utilizaram modelos e especificações distintas da aqui empregada. Comparado ao grupo de menor escolaridade, de modo geral, as chances de FP ou FN não apresentaram significância estatística. $\mathrm{Na}$ Índia, Onur e Velamuri ${ }^{28}$ observam que níveis mais altos de escolaridade diminuem a propensão ao registro de FN.

A diminuição das chances de falso positivo à medida que se aumenta o tempo desde a última consulta provavelmente relaciona-se com uma baixa capacidade de compreensão dos diagnósticos por parte dos pacientes. Velakkal et al. ${ }^{4}$ destacam que um dos fatores por trás da discordância entre as medidas autorreferidas e as laboratoriais é, muitas vezes, a incapacidade de compreensão do diagnóstico recebido. Indivíduos mais vulneráveis (mais velhos, menos escolarizados) apresentam níveis mais baixos de conhecimento relacionado à própria saúde.

Convém destacar que os casos de falso positivo (e a especificidade) podem ser artificialmente influenciados pelos casos em que os indivíduos apresentam a doença controlada por meio de medicamento. Nesses casos, o diagnóstico autorreportado será positivo (corretamente), mas a medida laboratorial apontará para outra direção em função do controle da enfermidade. Da mesma forma, algum exame prévio pode ter dado resultado positivo em outro momento da vida e, mesmo que não esteja mais com parâmetros alterados, o indivíduo pode reportar a presença da doença. No caso da diabetes, a base de dados nos permitiu excluir da análise os casos em que a diabetes poderia estar controlada com base no uso de medicamentos. No entanto, para os demais desfechos, o mesmo exercício não pôde ser realizado. Outra situação que deve ser levada em conta é a possibilidade de erros no exame realizado para a PNS, assim como potencias erros em outros exames prévios. Nos serviços de saúde, de modo geral, é prática comum a repetição de exames para dirimir dúvidas.

O presente trabalho apresenta limitações importantes. O período decorrido entre a coleta das duas informações de, em média, dois anos, entre a primeira e a segunda etapas da PNS, sem dúvida eleva as chances de discordância entre as medidas em função da eventual mudança das condições de saúde dos indivíduos. Outra limitação já mencionada é que não foram considerados nas análises os casos em que as doenças (colesterol e DRC) possam estar controladas em função da utilização de medicamentos. É provável que casos de pessoas que declararam ter recebido o diagnóstico médico, mas que apresentam a situação controlada por meio de medicamentos, tenham sido consideradas como falso positivo.

\section{Conclusão}

O estudo apontou a baixa sensibilidade da morbidade autorreferida para a detecção de doenças, em especial para a renal crônica e colesterol alterado. As medidas autorreferidas podem ter subestimado a prevalência das doenças, mostrando que os inquéritos que utilizam apenas medidas autorreportadas devem ser analisadas com algum nível de cautela. Assim, diante da viabilidade, recomenda-se que os componentes laboratoriais sejam empregados de rotina nos inquéritos populacionais para estimar a prevalência das doenças na população brasileira. Os resultados do presente trabalho apontam que o uso de serviços de saúde foi um importante determinante da capacidade da população em informar corretamente a sua condição de saúde, contribuindo no correto monitoramento e cuidado com a doença. 


\section{Colaboradores}

PC Pinheiro, MBA Barros, CL Szwarcwald, IE Machado, DC Malta conceberam e projetaram o estudo. PC Pinheiro desenvolveu a gestão, exploração e análise dos dados, a elaboração e interpretação dos resultados e a discussão. Todos os autores revisaram criticamente o manuscrito e colaboraram em todo o processo. Todos os autores leram, contribuíram para e aprovaram o manuscrito final.

\section{Referências}

1. Moreira JPL, Almeida RMVR, Luiz RR. Correção da prevalência autorreferida em estudos epidemiológicos com grandes amostras. Cad Saude Publica 2016; 32(12):e00050816.

2. Johnston DW, Propper C, Shields MA. Comparing subjective and objective measures of health: Evidence from hypertension for the income/health gradient. $J$ Health Econ 2009; 28(3):540-552.

3. Davillas A, Jones AM, Benzeval M. The Income-Health Gradient: Evidence From Self-Reported Health and Biomarkers in Understanding Society. In: Panel Data Econometrics: Empirical Applications. Amsterdam: Elsevier; 2019. p. 709-741.

4. Velakkal S, Subramanian SV, Millet C, Basu S, Stucker D, Ebrahim S. Socioeconomic Inequalities in NonCommunicable Diseases Prevalence in India: Disparities between Self- Reported Diagnoses and Standardized Measures. PLoS One 2013; 8(7):e68219.

5. Malta DC, Duncan BB, Schmidt MI, Machado IE, Silva AG, Bernal RTI, Pereira CA, Damacena GN, Stopa SR, Rosenfeld, LG, Szwarcwald CL. Prevalência de diabetes mellitus determinada pela hemoglobina glicada na população adulta brasileira, Pesquisa Nacional de Saúde. Rev Bras Epidemiol 2019; 22(Supl. 2):E190006.

6. Malta DC, Machado IE, Pereira CA, Figueiredo AW, Aguiar LK, Almeida WS, Souza MFM, Rosenfeld LG, Szwarcwald CL. Avaliação da função renal na população adulta brasileira, segundo critérios laboratoriais da Pesquisa Nacional de Saúde. Rev Bras Epidemiol 2019; 22(Supl. 2):E190010.

7. Malta DC, Szwarcwald CL, Machado IE, Pereira CA, Figueiredo AW, ACMGN Sá, Velasquez-Melendez G, Santo FM, PB Souza Junior, Stopa SR, Rosenfeld LG. Prevalência de colesterol total e frações alterados na população adulta brasileira: Pesquisa Nacional de Saúde. Rev Bras Epidemiol 2019; 22(Supl. 2):E190005.

8. Barros MBA, Francisco PMSB, Zanchetta LM, César CLC. Tendências das desigualdades sociais e demográficas na prevalência de doenças crônicas no Brasil, PNAD: 2003- 2008. Cien Saude Colet 2011; 16(9):3755-3768.

9. Skkiner K, Miller DR, Lincoln E, Lee A, Kazis LE. Concordance Between Respondent Self-reports and Medical Records for Chronic Conditions Experience From the Veterans Health Study. J Ambulatory Care Manage 2005; 28(2):102-110.

10. Okura Y, Urban LH, Mahoney DW, Jacobsen SJ, Rodeheffer RJ. Agreement between self-reported questionnaires and medical record data was substantial for diabetes, hypertension, myocardial infarction and stroke but not for heart failure. J Clin Epidemiol 2004; 57(10):1096-1103.

11. Szwarcwald CL, Malta DC, Souza-Júnior PRB, Almeida WS, Damacena GN, Pereira CA, Rosenfeld LG. Exames laboratoriais da Pesquisa Nacional de Saúde: metodologia de amostragem, coleta e análise dos dados. Rev Bras Epidemiol 2019; 22(Supl. 2):E190004. 
12. Mill JG, Malta DC, Machado IE, Pate A, Pereira CA Jaime PA, Szwarcwald CL, Rosenfeld LG. Estimativa do consumo de sal pela população brasileira: resultado da Pesquisa Nacional de Saúde 2013. Rev Bras Epidemiol 2019; 22(Supl. 2):E190009.

13. Souza-Júnior PR, Freitas MPS, Antonaci GA, Szwarcwald CL. Desenho da amostra da Pesquisa Nacional de Saúde 2013. Epidemiol Serv Saúde 2015; 24(2):207216.

14. Kidney Disease Improving Global Outcomes. KDIGO 2012 Clinical Practice Guideline for the Evaluation and Management of Chronic Kidney Disease. Kidney Int Supl 2013 [acessado 2020 Ago 6]; 3(1):1-150. Disponível em: https://kdigo.org/wp-content/uploads/2017/02/KDIGO_2012_CKD_GL.pdf

15. Faludi AA, Izar MCO, Saraiva JFK, Chacra APM, Bianco HT, Afiune Neto A, Bertolami A, Pereira AC, Lottenberg AM, Sposito AC, Chagas ACP, Casella-Filho A, Simão AF, Alencar Filho AC, Caramelli B, Magalhães CC, Magnoni D, Negrão CE, Ferreira CES, Scherr C, Feio CMA, Kovacs C, Araújo DB, Calderaro D, Gualandro DM, Mello Junior EP, Alexandre ERG, Sato IE, Moriguchi EH, Rached FH, Santos FC, Cesena FHY, Fonseca FAH, Fonseca HAR, Xavier HT, Pimentel IC, Giuliano ICB, Issa JS, Diament J, Pesquero JB, Santos JE, Faria Neto JR, Melo Filho JX, Kato JT, Torres KP, Bertolami MC, Assad MHV, Miname MH, Scartezini M, Forti NA, Coelho OR, Maranhão RC, Santos Filho RD, Alves RJ, Cassani RL, Betti RTB, Carvalho T, Martinez TLR, Giraldez VZR, Salgado Filho W. Atualização da Diretriz Brasileira de Dislipidemias e Prevenção da Aterosclerose - 2017. Arq. Bras. Cardiol 2017 Aug [cited 2020 Aug 7]; 109(2 Supl. 1):1-76. Available from: http://www.scielo.br/scielo.php?script=sci_arttext\&pid=S0066-782X2017001100001\&lng=en

16. Ning $M$, Zhang $Q$, Yang $M$. Comparison of selfreported and biomedical data on hypertension and diabetes: findings from the China Health and Retirement Longitudinal Study (CHARLS). BMJ Open 2016; 6:e009836.

17. Crimmins EM, Vasunilashorn SM. Biodemography: Adding Biological Insight into Social, Economic, and Psychological Models of Population and Individual Health Change with Age. In: Kenneth F, Ferraro LKG, editors. George Handbook of Aging and the Social Sciences. Amsterdam: Elsevier; 2016. p. 55-75.

18. Harris KM, Schorpp KM. Integrating Biomarkers in Social Stratification and Health Research. Annu Rev Sociol 2018; 44:361-386.

19. Lima-Costa MF, Peixoto SV, Firmo JOA, Uchoa E. Validade do diabetes auto-referido e seus determinantes: evidências do projeto Bambuí. Rev Saude Publica 2007; 41(6):947-953.

20. Wu SC, Li CY, Ke DS. The agreement between self-reporting and clinical diagnosis for selected medical conditions among the elderly in Taiwan. Public Health 2000; 114(2):137-142.
21. Molenaar EA, Van Ameijden EJ, Grobbee DE, Numans ME. Comparison of routine care self-reported and biometrical data on hypertension and diabetes: results of the Utrecht Health Project. Eur J Public Health 2007; 17(2):199-205.

22. Goldman N, Lin I-F, Weinstein M, Lin Y-H. Evaluating the quality of self-reports of hypertension and diabetes. J Clin Epidemiol 2003; 56(2):148-154.

23. Stopa SR, Malta DC, Monteiro CN, Szwarcwald CL, Goldbaum M, Galvão CCL. Acesso e uso de serviços de saúde pela população brasileira, Pesquisa Nacional de Saúde 2013. Rev Saude Publica 2017; 51(Supl. 1):3s.

24. Chrestani MAD, Santos IS, Matijasevich AM. Hipertensão arterial sistêmica auto-referida: validação diagnóstica em estudo de base populacional. Cad Saude Publica 2009; 25(11):2395-2406.

25. Vargas CM, Burt VL, Gillum R, Pamuk ER. Validity of self-reported hypertension in the National Health and Nutrition Examination Survey III, 1988-1991. Prev Med 1997;26(5 Pt 1):678-685.

26. Lee HY. Socioeconomic Disparities in the Prevalence, Diagnosis, and Control of Hypertension in the Context of a Universal Health Insurance System. J Korean Med Sci 2017; 32(4):561-567.

27. Instituto Brasileiro de Geografia e Estatística (IBGE). Sintese de indicadores sociais: uma análise das condições de vida da população brasileira. Rio de Janeiro: IBGE; 2016.

28. Onur I, Velamuri M. The gap between self-reported and objective measures of disease status in India. PLoS One 2018; 13(8):e0202786.

Artigo apresentado em 02/12/2020

Aprovado em 02/12/2020

Versão final apresentada em 04/12/2020

Editores-chefes: Maria Cecília de Souza Minayo, Romeu Gomes, Antônio Augusto Moura da Silva 
